\title{
Geleitwort zur Jubiläumsausgabe
}

\author{
Heiko Maas
}

(C) Springer Fachmedien Wiesbaden GmbH, ein Teil von Springer Nature 2019

Das Schwungrad der Geschichte dreht mit unterschiedlichen Geschwindigkeiten. Das zeigen die zehn Jahre, in denen die Zeitschrift für Außen- und Sicherheitspolitik die deutsche Diskussion seit 2008 bereichert und belebt hat in ganz besonderer Weise. In den vergangenen Jahren haben wir immer wieder Ereignisse und Entwicklungen erlebt, die mit großem Tempo und Wucht daherkamen und uns wahrscheinlich noch lange beschäftigen werden: die völkerrechtswidrige Annexion der Krim durch Russland und der Ausbruch der Kämpfe in der Ostukraine, ein massiver und weitgehend unerwarteter Bruch der europäischen Ordnung. Wir erlebten kurze, von einem großen Freiheitswillen inspirierte Revolutionen in der arabischen Welt, die bisher nur in Tunesien zu einem Mehr an Demokratisierung geführt haben. In Syrien und Irak eroberte der sogenannte Islamische Staat weite Gebiete, in denen er mordete und wütete, während er gleichzeitig in Europa blutige Attentate verübte. Wir sahen die Entstehung eines weiten Krisenbogens, der von Syrien, über Jemen bis nach Mali und Nigeria reicht. Schließlich erlebten wir 2015 eine Migrationsbewegung bislang ungekannten Ausmaßes Richtung Europa, vor allem von Flüchtlingen aus den Krisengebieten in Syrien, Irak und Afghanistan. All diese und weitere Krisen und Konflikte, wie das Ringen um Nordkoreas Nuklearprogramm oder das Atomabkommen mit Iran, wirken bis heute.

Andere Entwicklungen, wie der weitere Aufstieg Chinas, die Krise der global governance und des Multilateralismus und die Auswirkungen von disruptiven Technologien, Künstlicher Intelligenz und Big Data sowie die Verfügungsgewalt darüber, werden vielleicht einmal als die eigentlichen Auslöser des aktuellen Epochenwechsels beschrieben werden. Ihr langfristiger Einfluss auf die Veränderungen des internationalen Systems und die internationalen Beziehungen ist derzeit nur zu erahnen.

H. Maas $(\bowtie)$

Auswärtiges Amt, Werderscher Markt 1, 10117 Berlin, Deutschland 
Er kann aber kaum zu groß, zu tiefgehend eingeschätzt werden, denn die neuen Kräfte beginnen erst nach und nach zu wirken.

Wo stehen wir heute? Diese Einschätzung wird allerdings vielfach ohne die aktive Mitwirkung der USA geschehen müssen. Denn die amerikanische Politik ist seit der Wahl von Donald Trump zum Präsidenten im November 2016 von Ambivalenz und Unberechenbarkeit gekennzeichnet. In der NATO erfahren die Europäer zwar einerseits eine weiterhin kräftige Unterstützung und Solidarität der USA, beispielsweise im Rahmen der European Deterrence Initiative. Andererseits zweifelt der USPräsident öffentlich und in fast spielerischer Manier am Wert einer Allianz, in der die finanziellen Lasten nicht fair verteilt seien. Im Handel befinden sich die USA mit der Europäischen Union und anderen Partnern im offen ausgetragenen Streit; Strafzölle wurden verhängt, die EU als Gegner bezeichnet. Das ist das Gegenteil jenes immer enger verzahnten transatlantischen Wirtschaftsraums, den wir noch vor zwei Jahren als gemeinsames Ziel hatten. Die unterschiedlichen Auffassungen über die Wirksamkeit des Atomabkommens mit dem Iran führen zu einer US-Sanktionspolitik, die europäische Firmen hart treffen kann. In dieser Lage ist es von strategischer Bedeutung, den USA zwei Dinge zu versichern: Wir wollen für die Sicherheit der Region eng zusammenarbeiten. Aber wir lassen es nicht zu, dass über unsere Köpfe hinweg zu unseren Lasten gehandelt wird.

Und schließlich haben wir das weite Feld der Multilateralen Organisationen und Rahmenwerke, einschließlich der Vereinten Nationen, an dem die USA nicht mehr interessiert zu sein scheinen. Der auch innerhalb der USA stark kritisierte Ausstieg aus dem Pariser Klimaabkommen kurz nach Regierungsantritt Trumps war nur der erste Spiegelstrich einer Agenda, die dadurch geprägt sein könnte, aus der Aufkündigung eines regelbasierten Miteinanders eine gewinnbringende Strategie zu entwickeln, in der Erwartung, durch direktes bilaterales Interagieren den eigenen Machtvorteil optimal ausspielen zu können.

Wir wissen nicht, wie sich diese Politik weiter entwickelt, ob sie eine langfristige Weichenstellung bedeutet oder nur ein vorübergehendes Phänomen beschreibt. Gleichzeitig lernen wir, was es für Europa und die Welt bedeutet, wenn sich die USA in manchen sicherheitspolitischen Dossiers auf dem Rückzug befinden oder gar nicht mehr präsent sind. Schon der ehemalige US-Präsident Barack Obama hatte ein Disengagement aus manchen Regionen angekündigt und mit dem Pivot to Asia eine Schwerpunktverlagerung von Europa und dem Nahen und Mittleren Osten in Richtung Asien-Pazifik angestrebt. In der Summe erweckt all dies den Anschein, dass das transatlantische Verhältnis die Balance verliert und neu gestaltet werden muss, um diese dauerhaft zu erhalten.

Es ist somit höchste Zeit, unsere Partnerschaft neu zu vermessen - nicht um sie hinter uns zu lassen, sondern um sie zu erneuern und zu bewahren. Als Bauplan dient uns die Idee einer balancierten Partnerschaft, in der wir unseren ausgewogenen Teil der Verantwortung übernehmen. In der wir dort Gegengewichte schaffen, wo die USA rote Linien überschreiten. In der wir unser Gewicht einbringen, wo sich Amerika zurückzieht. Und in der wir neu miteinander ins Gespräch kommen. In allen Fällen wird Europa mehr tun. 
Unsere Antworten: Deutschland stand während dieser Entwicklungen nicht abseits. Es hat die so oft eingeforderte Verantwortung übernommen und trägt sie auch heute. Das zeigt unser Engagement im Normandie-Format und im Minsk-Prozess zur Einhegung und möglichen Lösung des Konflikts in der Ostukraine, das zeigen unsere Milliarden für humanitäre Hilfe und die zahlreichen Projekte im Rahmen unserer Stabilisierungspolitik. Vielfach sind wir im Nahen Osten einer der größten Geldgeber weltweit. Auch die rund 3500 Soldaten in den Auslandseinsätzen und unser Beitrag zur NATO Enhanced Forward Presence im Baltikum sind ebenfalls Ausdruck dieser Verantwortung. Für die kommenden zwei Jahre wurden wir mit überwältigender Mehrheit von der Generalversammlung zum Mitglied des UNSicherheitsrats gewählt und haben hier zusätzliche Möglichkeiten, für unsere und europäische Interessen und Werte, vor allem aber für eine stabile und gerechte internationale Ordnung einzutreten.

Deutschland kann aber noch mehr tun, um auf die neuen großen Linien der internationalen Politik angemessen zu reagieren. Und wir werden mehr tun, denn im internationalen Maßstab sind wir ein starker, auch wohlhabender Akteur. Bei aller Bescheidenheit und gebotener Zurückhaltung dürfen wir uns nicht zu klein machen. Wie soll die EU Schlagkraft entwickeln, wenn nicht auch ihr wirtschaftlich stärkster und bevölkerungsreichster Staat mit mutigen Schritten vorangeht, um einen Unterschied in der internationalen Politik zu machen? Nur als eine Einheit, die ihre Souveränität bündelt - als Europe United - sind wir eine globale Größe. Nicht zuletzt, weil Europa das Recht des Schwächeren akzeptiert und aus seiner Geschichte weiß, dass Kooperation kein Nullsummenspiel ist. Ein Europa der Nationalstaaten dagegen wäre machtlos und kraftlos.

Bei der Bewältigung unserer Aufgaben ist die EU daher unser entscheidender Handlungsrahmen. Dass die EU dabei selbst Risse aufweist, vor allem, dass die Briten die EU verlassen wollen, ist eine zusätzliche Herausforderung, mit der wir umgehen müssen. Das Schrumpfen der traditionellen Parteien der Mitte, über Jahrzehnte Träger der europäischen Idee, und die Erfolge national-gesinnter, oft populistischer und anti-europäischer Parteien zeigen zudem, wie tief die gesellschaftlichen Verwerfungen reichen. Es sind auffällige Parallelen zu den USA, dass auch in Europa Finanz- und Wirtschaftskrise, Globalisierung und Migration zu Verunsicherungen geführt haben, die vielfach in einem Vertrauensverlust der Bürger in die traditionelle Politik und ihre Verfahren münden. Dennoch zeigen insbesondere die vergangenen zehn Jahre bis hin zum Sommer 2018, dass der Schlüssel zu den Lösungen eben nicht in einer nationalen Politik des My country first liegt. Weder für uns noch für andere Staaten der Welt.

Für ein souveränes und starkes Europa: Das gilt erst recht innerhalb Europas. Über die finanzielle Unterstützung Griechenlands und anderer Staaten ist hart gerungen worden, in jedem Land auch intern - nicht zuletzt in Deutschland. Die Zukunft und die Stabilität des Euros standen auf dem Spiel, aber auch die Lebens- und Zukunftsperspektive von Millionen Menschen. Im Ergebnis war Europa solidarisch und dies mit Erfolg: Nacheinander konnten Staaten wie Irland oder Portugal zu einer normalen Haushaltsführung zurückkehren. Sie verzeichnen heute Wirtschaftswachs- 
tum und sinkende Arbeitslosenzahlen. Selbst Griechenland ist inzwischen an die Finanzmärkte zurückgekehrt und erlebt einen wirtschaftlichen Aufschwung. Europa ist noch nicht wieder so stark wie es sein könnte, aber unsere Finanzsysteme sind heute krisenfester als früher.

Auch in der Außenpolitik haben wir in der EU gemeinsame Antworten gefunden und neue Instrumente geschaffen. Die völkerrechtswidrige Politik Russlands haben wir mit Geschlossenheit und einer wirksamen Sanktionspolitik beantwortet. Der Flüchtlingstragödie und der vielfachen Migration nach Europa begegnen wir mit einem breiten Paket an Maßnahmen wie humanitärer Hilfe in den Krisenregionen, Kapazitätsaufbau der dortigen Nachbarstaaten und Stärkung der Grenzschutzagentur Frontex. Unsere sicherheitspolitischen Bedürfnisse zu wahren, aber unseren Charakter als Kontinent der Humanität nicht zu verlieren, ist schon jetzt eine Herausforderung. Ihre Dimensionen werden vor allem aufgrund der demographischen Entwicklung aber noch beträchtlich zunehmen. Wir versuchen die Ursachen deshalb verstärkt in Afrika selbst anzugehen. Zu den zahlreichen, langfristig orientierten Maßnahmen gehören zum Beispiel der Aufbau und die Stärkung des Sicherheitssektors in Subsahara-Afrika durch die EU. Es ist entscheidend, dass sich Staaten gegen islamistische Terroristen, organisierte Kriminalität oder radikale Milizen wehren können, damit bewaffnete Konflikte als Fluchtursache eingedämmt werden. Die EU-Trainingsmission in Mali, sowie die gleichzeitige UN-Mission MINUSMA, aber auch die Unterstützung der fünf Sahelnationen Burkina Faso, Niger, Mauretanien, Mali und Tschad bei ihrer gemeinsamen Einsatzgruppe veranschaulichen das.

Wir sind uns bewusst, dass wir sicherheitspolitisch noch handlungsfähiger werden müssen. Daher brauchen wir einen starken Europäischen Pfeiler in der NATO, als Ausdruck unserer Souveränität, aber auch als Zeichen, dass sich Amerika auf uns verlassen kann. Solch eine Europäische Sicherheits- und Verteidigungsunion erfordert, dass auch die Entwicklungen im Rahmen der militärischen Säulen der Gemeinsamen Sicherheits- und Verteidigungspolitik der EU (GSVP) zügig entscheidende Fortschritte bringen; ebenso wie die Zusammenarbeit zwischen der EU und der NATO. Seit der Verabschiedung der Globalen Strategie der EU im Jahr 2016 haben wir weit mehr erreicht, als in vielen Jahren zuvor. Gleiches muss nun auch für die zivile GSVP gelten, wo wir den Aufbau eines Europäischen Stabilisierungscorps vorantreiben. Schließlich benötigen wir schnell verfügbare multinationale Expertenteams aus vielen Bereichen (unter anderem Polizei, Juristen ${ }^{1}$, Verwaltungsexperten, Techniker, Mediziner), die fertig ausgebildet und trainiert ohne großen Vorlauf in Einsätze gehen können. Gemeinsam mit dem Zentrum für Internationale Friedenseinsätze arbeiten wir an einer Europäisierung dieser Idee.

Diese Elemente und Instrumente müssen allesamt Teil einer selbstbewussten europäischen Politik werden, die in den kommenden Jahren tragfähige Antworten auf die Frage findet, wie sich Europa als ein starker Pol in einer neuen multipolaren Weltordnung behaupten kann. Dies schließt in Handelsfragen eine Widerstandsfähigkeit gegen andere, auch gegen eine feindliche US-Politik, ein. Wenn unsere Wirtschaft

\footnotetext{
1 Im vorliegenden Artikel wird, abweichend vom ZfAS-Standard, bei personenbezogenen Substantiven die herkömmliche grammatikalische Form verwendet. Der Autor schließt damit selbstverständlich alle Personen des Berufsstandes ein.
} 
unabgestimmt und nicht regelkonform Opfer von unspezifischer Sanktionspolitik gegen Drittstaaten wird - wie es bei China, Russland oder dem Iran vorkommt müssen wir uns mit einer souveränen Handels-, Finanz- und Wirtschaftspolitik effektiv schützen können.

Aus meiner Sicht benötigen wir ein großes und integratives Europa, das sich nicht spalten lässt, sondern die Gegensätze und Konflikte zwischen Nord und Süd, Ost und West überwindet. Am Anfang muss dabei die aktivere Gestaltung des eigenen Umfeldes stehen: die östliche Nachbarschaftspolitik und das künftige Verhältnis zu Russland, gegenüber unseren Partnern auf dem Westbalkan mit ihren Mitgliedschaftsperspektiven für NATO und EU, in Bezug auf den Nahen und Mittleren Osten, einer Region, die uns betrifft, in der wir aber derzeit zu wenig Einfluss haben, und bezüglich der europäischen Gegenküste, dem afrikanischen Kontinent. Deutschland ist sich mit Frankreich und anderen Staaten einig, dass wir Europäer stärker werden müssen, um wirklich mitgestalten zu können. Wenn Europa diesen Gestaltungsanspruch aufgäbe oder nicht umsetzte, würde es aufhören, Akteur zu sein. In Abwesenheit der USA würden andere, wie Russland und China, an unsere Stelle treten mit eigenen Interessen, die wir selten teilen. Für die Zukunft Europas und die Selbstbestimmung seiner Bürger kann dies keine Option sein - schließlich geht es hier um unsere Unabhängigkeit und Sicherheit. Es geht um ein souveränes Europa!

Für uns gilt: Multilateralismus first! Deshalb wird die Zukunft auch global mehr von uns und all jenen fordern, die ein fundamentales Interesse an einer Stärkung des Multilateralismus haben. Wenn sich die USA hier dauerhaft zurückziehen, bröckelt das Fundament des internationalen Systems. Und manche aufstrebende Macht, die das westlich geprägte internationale Normen- und Regelsystem eher als Korsett denn als Garant für Frieden, Stabilität und Fairness im internationalen Staatensystem empfindet, sieht die Chance, die Welt nach ihren Vorstellungen und Vorteilen zu formen. Es bedarf daher einer Allianz für den Multilateralismus. Nicht als Allianz gegen irgendjemanden, sondern als Netzwerk gleichgesinnter Staaten für gemeinschaftliche Lösungen globaler Probleme. Dazu zählt der Klimawandel, der zunehmende Protektionismus in Handelsfragen, die Rüstungskontrolle oder der Umgang mit der weltweiten Flüchtlingskrise. Es geht um ein Bündnis all jener, denen Regeln und Völkerrecht, Fairness und Kooperation in der internationalen Politik wichtig ist. Es geht um Gleichgesinnte, die die bestehenden Institutionen gemeinsam stärken wollen - anstatt ihre Schwächung hinzunehmen. Dies gilt vor allem in Zeiten schneller Umbrüche und Veränderungen, in denen viele außenpolitische Selbstverständlichkeiten der Vergangenheit angehören. In Zeiten, wie wir sie in den vergangenen zehn Jahren erlebt haben; in Zeiten, wie wir sie in den kommenden zehn Jahren sicherlich erleben werden. Die Tür steht hier allen offen, die ähnlich denken - das Engagement der USA ist ausdrücklich erwünscht!

Ich bin überzeugt, die Zeitschrift für Außen- und Sicherheitspolitik und ihre Autoren werden diese Epoche weiterhin kenntnisreich kommentieren und analysieren. Sie werden dabei auch die Arbeit der Bundesregierung und des Auswärtigen Amts kritisch begleiten und uns manche Anregung geben. Das ist hoch willkommen, denn 
der Austausch zwischen Wissenschaft und Praxis ist gerade in volatilen Zeiten von besonderem Wert.

Heiko Maas

Außenminister der Bundesrepublik Deutschland 